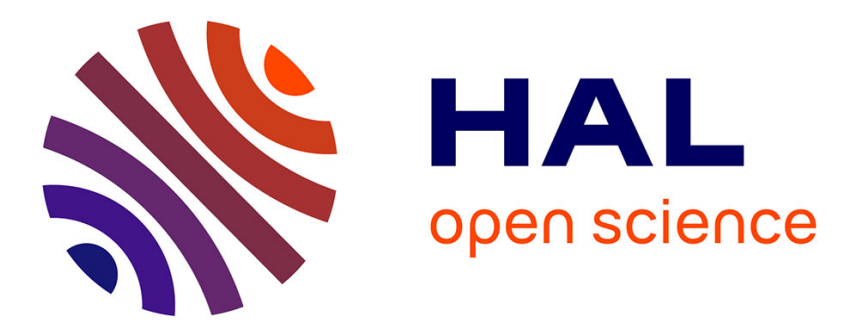

\title{
Microrobotic simulator for assisted biological cell injection
}

\author{
Hamid Ladjal, Jean-Luc Hanus, Antoine Ferreira
}

\section{To cite this version:}

Hamid Ladjal, Jean-Luc Hanus, Antoine Ferreira. Microrobotic simulator for assisted biological cell injection. IEEE International Conference on Robotics and Automation, Sep 2011, San Francisco, United States. pp.1315 - 1320, 10.1109/IROS.2011.6094965 . hal-00657611

\section{HAL Id: hal-00657611 https://hal.science/hal-00657611}

Submitted on 7 Jan 2012

HAL is a multi-disciplinary open access archive for the deposit and dissemination of scientific research documents, whether they are published or not. The documents may come from teaching and research institutions in France or abroad, or from public or private research centers.
L'archive ouverte pluridisciplinaire HAL, est destinée au dépôt et à la diffusion de documents scientifiques de niveau recherche, publiés ou non, émanant des établissements d'enseignement et de recherche français ou étrangers, des laboratoires publics ou privés. 


\title{
Microrobotic Simulator for Assisted Biological Cell Injection
}

\author{
Hamid Ladjal ${ }^{\dagger}$, Jean-Luc Hanus*, Antoine Ferreira* \\ ${ }^{\dagger}$ LIRIS CNRS UMR 5205, Université Claude Bernard Lyon 1, France \\ Email: hamid.ladjal@liris.cnrs.fr \\ *Laboratoire PRISME, ENSI Bourges, France \\ Email: jean-luc.hanus, antoine.ferreira @ensi-bourges.fr
}

\begin{abstract}
This paper presents a complete computer-based biological training system to simulate intracytoplasmic sperm injection (ICSI) procedures in virtual environments (VEs). Using the proposed system, the user can practice and improve its control by developing a gesture similar to that performed in reality. In order to minimize the risk of damaging membranes during the injection, the needle must be removed quickly and safely. The human-machine user's interface provides an efficient haptic-based guidance tool. The key challenge of deformable simulation is to satisfy the conflicting requirements of real-time interactivity and physical realism. The compromise between the realism of bio-mechanical models used, the accuracy and stability of computational algorithms for real-time haptic rendering are fullfilled with the use of a mechanical modeling based on an hyperelastic model (St-Venant-Kirchhoff) and the writing of a specific dynamic finite element code. Simulated responses are compared to experimental data. These comparisons show the effectiveness of the proposed physically-based model.
\end{abstract}

Index Terms-micro-injection ICSI, finite element, modeling, real-time interaction, haptic feedback.

\section{INTRODUCTION}

Cell manipulation is a prevalent process in the field of molecular biology. This process plays an important role in intracytoplasmic sperm injection (ICSI), pronuclei deoxyribonucleic acid (DNA) injection, therapeutic and regenerative medicine, and other biomedical areas. ICSI has progressively replaced all other micro-injection procedures for overcoming intractable male-factor infertility and has emerged in a relatively short time as a routine procedure for many in-vitro procedures. Nevertheless, injections are currently performed manually and technicians are required to be skillful enough to not destruct the cell structure of the ovum during the ICSI process. As a consequence, the success rate remains relatively low and strongly dependant on the experience and skills of the operator [1], [2], [3]. Various cell injection systems have been developed to provide more controllable manipulation of biological cells [23], [24]. If visual servoing is a necessary condition for precise micro-injection operations, the knowledge of interactive forces acting during pipette insertion plays an important role too since it can be used to provide force feedback for precise regulation of needle penetration speed and stength [4]. Reducing cell deformation and needle deflection will also lower the risk of cell damage. However, when manipulating deformable biological objects, force sensor measurement will provide only the local forces at the pipette puncture point which limits strongly the operator haptic rendering [4]. In order to learn, train and analyze basic cell injection procedures, most operators are aiming to use artificial cells because practicing on human oocyte has ethical concerns and potential risks [13]. The development of computer-based systems using visual and force-feedback for both injection training and injection assistance will help to overcome some of these problems. Similar research on virtual reality environments platforms has been carried out at a macroscopic scale in surgery simulation and modeling of soft tissues [5], [6], [7]. Needle punctures and passes through different tissue layers such as skin, muscle, fatty and connective tissue have been modeled [8], [9], [26], [27]. By analogy to in vivo intracytoplasmic sperm injection, similar boundary conditions occur. Within the framework of microinjection, the cell geometry and bio-mechanical subcomponents properties (biomembrane, cytoskeleton, cytoplasm, nucleus) are of major importance in simulation and modeling because these factors affect the amount of cell membrane deformation, needle deflection and interaction forces [10], [11]. A nonlinear bio-mechanical cell model is required to render in a realistic way the interactions between the needle and the cell for accurate insertion [12].

The objective of this paper is to develop and implement a bio-mechanical finite element approach dedicated to real-time cell injection to facilitate training of ICSI operations. We developed two models based either on an explicit linear finite element model or on a non-linear finite element Saint-VenantKirchooff material. These models include the topological information of the living cells (shape and dimensions), and the biological structure (cytoplasm layers, cytoskeletons and nucleus) [13], [14], [15].

This paper is organized as follows: in Section II, we introduce the main requirements for ICSI trainers. In Section III, we present the bio-mechanical finite element approach dedicated to real-time injection. In Section IV, we present the methodology of the virtual environment system for cell injection. In Section V, we make a comparative study between our real-time cell injection simulator and experimental data. Finally, we give some concluding remarks and the directions for future work.

\section{INTRACYTOPLASMIC SPERM INJECTION TRAINERS}

The intracytoplasmic sperm injection (ICSI) is one of the most important advances in assisted reproductive technology 
in recent years. ICSI is considered a breakthrough in the treatment of male infertility issues. Conventional methods of ICSI injection require the operator to undergo long training (over a period of one year), and the success rates are low (around 10\% - 15\%) due to poor reproducibility. The fragile nature of a biological cell requires the operator to be efficient; otherwise the patrician may damage the cell. There are also chances of contamination due to direct human involvement. The drawbacks involved in conventional methods have motivated the research community to develop robotic-based tools to perform cell injection tasks. One of the main features of these systems is to have better control on the movement of the micro-pipette used for injecting cells.

In order to facilitate training of biological cell injection operations, we have developed and implemented an interactive virtual tool for clinical and ethical formation. The operator improves his gesture in a similar way to that carried out in reality without involving ovocyte tests coming from animals or humans. The design of such an environment of simulation requires to satisfy the conflicting requirements of realtime interactivity and physical realism. A key challenge of deformable simulation is to find a compromise between the complexity of the realistic models and the computing speed. The proposed physics-based simulator aims to accurately restore bio-mechanical characteristics of the micro-injection task in a three-dimensional virtual environment.

\section{BIOMECHANICAL MODELS FOR CELL INJECTION}

We implemented and compared two models: the linear explicite finite element model and the hyperelastic model.

In this section, we present the numerical development of the linear elastic mass-tensor model [16], [17], and its extension to non-linear elasticity [18] with tetrahedral linear elements.

For an isotropic elastic or St Venant-Kirchhoff hyperelastic material the elastic energy, noted $W$, can be written as:

$$
W(\mathbf{E})=\frac{\lambda}{2}(\operatorname{tr} \mathbf{E})^{2}+\mu \operatorname{tr}\left(\mathbf{E}^{2}\right)
$$

where $\mathbf{E}$ is the Green-Lagrange strain tensor. A displacementbased finite element solution is obtained with the use of the principe of virtual works [19]. Using finite element method (FEM) notations, inside each tetrahedron $T^{k}$, the displacement field is defined by a linear interpolation $\left[\mathbf{N}^{\mathrm{k}}\right]$ of the nodal displacement vector $\left\{\mathbf{u}^{\mathbf{k}}\right\}$ of the four vertices of tetrahedron:

$$
\left\{\mathbf{U}(\mathbf{x})^{\mathbf{k}}\right\}=\left[\mathbf{N}^{\mathbf{k}}(\mathbf{x})\right] \quad\left\{\mathbf{u}^{\mathbf{k}}\right\}
$$

\section{A. Linear elastic finite element model}

In this section, we introduce the fundamental concepts for description and measurement of the deformation using finite element. For small deformations, the Green-Lagrange strain tensor is linearized into the infinitesimal strain tensor. The elastic energy is only a quadratic function of the displacement field. The elementary submatrices connecting the elementary force acting on the node $i$, to the displacement of the node $j$ are:

$$
\begin{aligned}
{\left[\mathbf{K}_{\mathbf{i j}}^{\mathbf{k}}\right]=} & \frac{1}{36 V^{k}}\left(\lambda\left\{\mathbf{m}_{\mathbf{i}}\right\}\left\{\mathbf{m}_{\mathbf{j}}\right\}^{T}+\right. \\
& \left.\mu\left\{\mathbf{m}_{\mathbf{j}}\right\}\left\{\mathbf{m}_{\mathbf{i}}\right\}^{T}+\mu\left\{\mathbf{m}_{\mathbf{i}}\right\}^{T}\left\{\mathbf{m}_{\mathbf{j}}\right\}[\mathbf{I}]\right)
\end{aligned}
$$

where $\{\mathbf{m}\}$ are unit outward-pointing normals to triangular faces and $V^{k}$ is the volume of the tetrahedron $T^{k}$. Taking into account the contribution of all adjacent tetrahedra, the global internal force acting on a node $l$ can be expressed as follows:

$$
\left\{\mathbf{F}_{\text {int }}^{\mathbf{l}}\right\}=\sum_{k \in \mathcal{V}_{l}}\left(\sum_{j=1}^{4}\left[\mathbf{K}_{\mathbf{i j}}^{\mathbf{k}}\right]\left\{\mathbf{u}_{\mathbf{j}}\right\}\right)
$$

where $\mathcal{V}_{l}$ is the neighborhood of vertex $l$ (i.e. the tetrahedra containing node $l$ ).

\section{B. Hyperelastic finite element model}

The simplest hyperelastic material model is the Saint VenantKirchhoff model which is just an extension of the linear elastic material model to the nonlinear regime. The algorithm is built in the same way. Since the strain is now quadratic in displacement, the elastic energy is a fourth-order polynomial in displacement. The relation between nodal forces and nodal displacement is non-linear and can be written as:

$$
\begin{aligned}
& \left\{\mathbf{F}_{\mathbf{i}}^{\tau}\right\}=\underbrace{\sum_{j=1}^{4}\left[\mathbf{B}_{\mathbf{i j}}^{\tau}\right]\left\{\mathbf{u}_{\mathbf{j}}\right\}}_{F_{1}^{\tau}} \\
& +\underbrace{\sum_{j, k=1}^{4}\left\{\mathbf{u}_{\mathbf{k}}\right\}\left\{\mathbf{u}_{\mathbf{j}}\right\}^{T}\left\{\mathbf{C}_{\mathbf{j k i}}\right\}+\frac{1}{2}\left(\left\{\mathbf{u}_{\mathbf{j}}\right\}^{T}\left\{\mathbf{u}_{\mathbf{k}}\right\}\right)\left\{\mathbf{C}_{\mathbf{i j k}}\right\}}_{F_{2}^{\tau}} \\
& +\underbrace{2 \sum_{j, k, l=0}^{3} D_{j k l i}\left\{\mathbf{u}_{\mathbf{l}}\right\}\left\{\mathbf{u}_{\mathbf{k}}\right\}^{T}\left\{\mathbf{u}_{\mathbf{j}}\right\}}_{F_{3}^{\tau}}
\end{aligned}
$$

where the global force can be written as:

$$
F_{i}^{T}=\underbrace{F_{1}^{T}}_{\text {Linearforce }}+\underbrace{F_{2}^{T}+F_{3}^{T}}_{\text {non-linearforce }}
$$

where $\left[\mathbf{B}_{\mathbf{i j}}\right],\left\{\mathbf{C}_{\mathbf{i j k}}\right\}, D_{i j k l}$ are respectively a matrix, a vector and a scalar. These tensors, depending on the remaining geometry and Lame's coefficients are constant. They can be pre-computed in an off-line phase. This is the essential advantage of the mass-tensor approach which makes it useful for real-time application.

\section{Dynamic Model}

The deformation of the deformable object is given by the displacement of the nodes according to the acting external and internal forces. In an interactive simulation the applied forces change in time and the virtual objects have to react to them in 
real time. Therefore, the FEM has to be simulated dynamically. The equation of motion of a vertex $l$ of the cell mesh can be written:

$M^{l}\left\{\ddot{\mathbf{u}}_{l}\right\}+\gamma^{l}\left\{\dot{\mathbf{u}}_{l}\right\}+\sum_{k \in \mathcal{V}_{l}}\left(\sum_{j=1}^{4}\left[\mathbf{K}_{\mathbf{i j}}^{\mathbf{k}}\right]\left\{\mathbf{u}_{\mathbf{j}}\right\}\right)=\left\{\mathbf{F}_{\mathbf{e x t}}^{\mathbf{l}}\right\}$

where $M^{l}$ and $\gamma^{l}$ are respectively the mass and damping coefficients of each vertex.

To solve the dynamic system, we tested different integration schemes (implicit and explicit) taking into account the tradeoff between real-time simulation and haptic stability requirements. We choose the explicit centered finite-difference scheme.

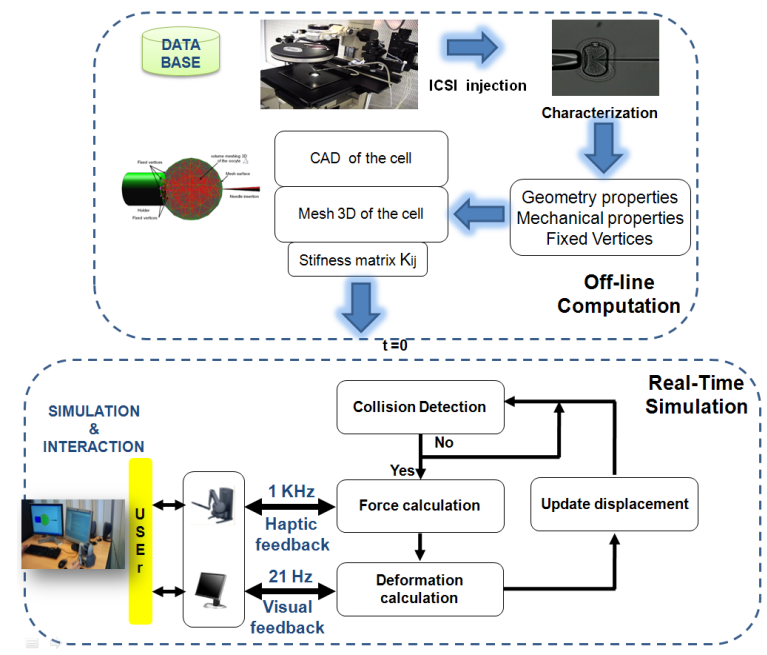

Fig. 1. Computational architecture for simulating force-reflecting deformable cell micro-injection in a virtual environment. The figure shows the two simulation phases used for the real-time micro-injection of the cell: (i) off-line pre-calculations of stiffness matrices and (ii) simulation of visual and haptic interaction.

\section{3D REAL-TIME VIRTUAL REALITY BASED ICSI SIMULA- TOR}

Real time virtual reality based cell simulation system, the operator would be able to interact with the threedimensional model of cell using his sense of vision as well as actively manipulate using his sense of touch. Fig. 1 shows the architecture of the real-time virtual reality based cell ICSI simulator. This includes the computer generated mesh of oocyte, the needle, collision detection algorithm, physical-based models of deformable cell modeling and haptic interaction controller. The operator is able to interact with the three-dimensional (3-D) model of the cell using his sense of vision as well as actively manipulate using his sense of touch. Usually, realtime graphics translates to an update rate of $25 \mathrm{~Hz}$, stable haptic interaction in virtual environments (VE's) requires much higher update rate of around $1 \mathrm{kHz}$. A significant difficulty of using the finite element technique for real-time simulation is most computationally costly, these mesh-based schemes require expensive numerical integration operation for the computation of the system stiffness matrices. We adopted a computational architecture with two simulation stages: (i) an off-line computation of stiffness matrices for each triangular element and (ii) visual and haptic interaction for real-time ICSI simulation.

\section{A. Off-line computation}

The proposed computational methodology is composed of an off-line pre-calculation step. The most costly and timeconsuming operations are realized during this step. The database contains geometric properties of oocyte cells using the micro-injection setup image analysis. The design geometric of oocyte cell models are based on a commercial computeraided design (CAD) package (MARC-ADAMS). The meshing of the $3 \mathrm{D}$ internal structure is then carried out through a dedicated 3D meshing software (GID software) modeled in exact dimensions. Although they were displayed as 3D texturemapped objects to the user, they were modeled as connected line segments to reduce the number of collision computations during real-time interactions.

The oocyte cell was approximated as an assembly of discrete tetrahedral elements interconnected to each other through a fixed number of nodes (see Fig.2). The displacements of these nodal points for applied external injection forces were the basic unknowns of our FEM analysis. The coordinates of vertices, the tetrahedron indexing, and the connectivity of vertices were derived from the geometric model. Then, the mechanical (young modulus, density) and geometrical (diameter, volume) properties of the cell structure are determined by a real tests carried out on a mouse oocyte [20].

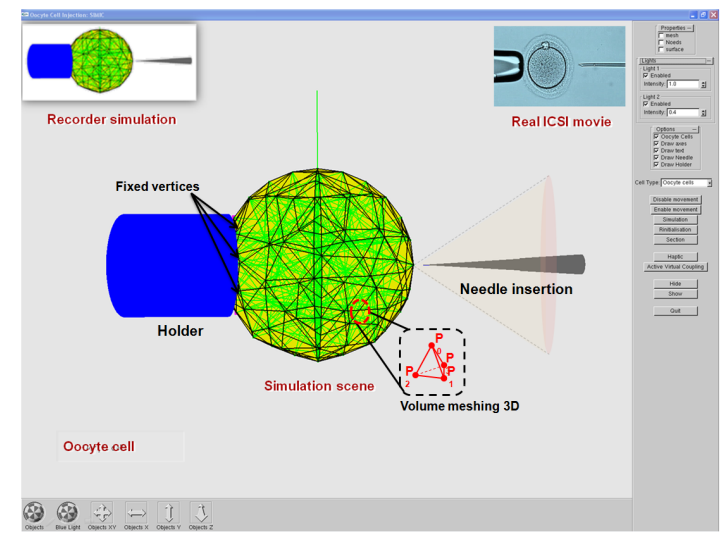

Fig. 2. Virtual graphic of the ICSI environnement: the 3D tetrahedral mesh of the mouse oocyte cell.

Finally, these properties are injected in the FEM algorithm for calculating the physically-based behavior of biological soft tissues. The off-line calculation of all tensors $K_{i j}$ allows precalculation stiffness values for preselected cells. The stiffness tensors $K_{i j}$ should be calculated for a set of oocyte parameters.

\section{B. Real-time haptics-enable simulator}

Since haptics and graphics have different update frequencies, we implemented separate threads to update the loops. During the injection task, the contact between the tip and the cell must occur at a special set of points (nodal points). The collision detection module specifies the type of tool-tissue 
interaction. The tool in a virtual environment can be modeled as a complex 3D object, composed of numerous surfaces, edges and vertices. However, due its structure complexity, realtime interaction is very challenging. In our implementation, we used a point based representation of the pipette injector and employed a simple ray-triangle intersection with local search technique [21]. To optimize the collision detection algorithm and cell deformation, we refine our mesh only in a puncture area. The mesh refinement is carried out in the precomputational process. The visual feedback is updated after each displacement calculation step and the haptic feedback is returned after each force calculation step.

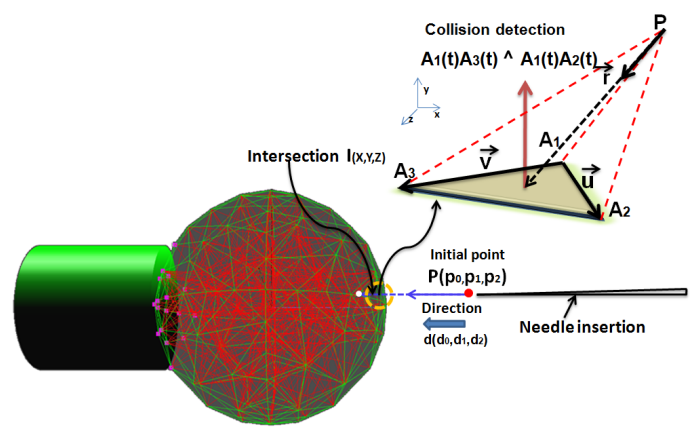

Fig. 3. Scheme illustrating the collision detection algorithm using a simple triangle points intersection problem: needle intersection with triangle surface of the cell, and the refining of the mesh.

1) Virtual coupling for stability of the haptic rendering: The internal operating loop of the haptic interface requires an update frequency around $1 \mathrm{KHz}$. However, the finite element model update frequency is in the range of $25-30 \mathrm{~Hz}$. This frequency difference threatens the coherence between both systems leading to instabilities of the user haptic rendering. We adopted the solution to use a virtual coupling model defined in [22]. This approach introduces a virtual passive link between the simulation model and the haptic interface in order to ensure the stability and the performance of the system Fig.(4.a). When we combine the impedance display implementation with an appropriate virtual coupling network, we get the admittance matrix for the combined interface. The linear two-port is said to be absolutely stable if exists no set of passive terminating one-port impedances for which the system is unstable. Llewellyn's stability criteria [25] provides both necessary and sufficient conditions for absolute stability of linear two-ports. We get the conditions for absolute stability of the haptic interface:

$$
\begin{gathered}
\operatorname{Re}\left(Z_{d i}(z)\right) \geq 0, \quad \frac{1}{Z_{c v i}(z)} \geq 0 \\
\cos (\angle Z O H(z))+\frac{2 \operatorname{Re}\left(Z_{d i}(z)\right) \operatorname{Re}\left(\frac{1}{Z_{c v i}(z)}\right)}{|Z O H(z)|} \geq 1
\end{gathered}
$$

Where $Z_{c v i}(z)$ is the virtual coupling impedance $\left(k_{c}, b_{c}\right)$, $Z O H(z)$ is a zero order holder, and $Z_{d i}(z)$ is the PHANToM impedance. The inequality (9) can be rewritten to get an explicit expression of absolute stability of the haptic interface:

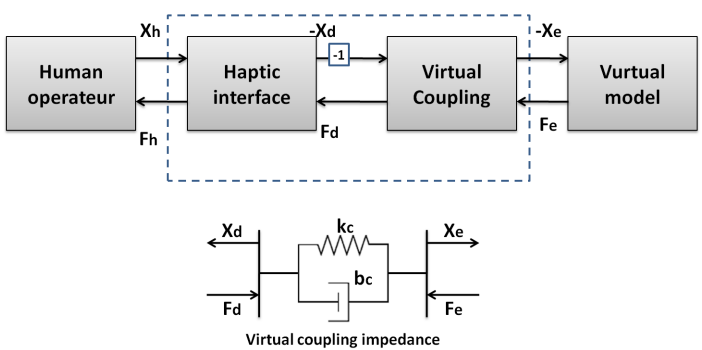

(a)

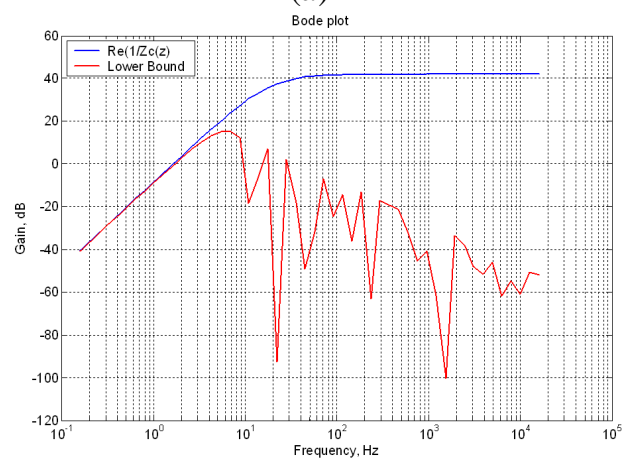

(b)

Fig. 4. Stability conditions using the virtual coupling model. (a); The model of virtual coupling, (b); Impedance display virtual coupling

$$
\operatorname{Re}\left(\frac{1}{Z_{c v i}(z)}\right) \geq \frac{1-\cos (\angle Z O H(z))}{2 \operatorname{Re}\left(Z_{d i}(z)\right)}|Z O H(z)|
$$

For the virtual coupling, the impedance display induces a limit on the maximum impedance which can be rendered. We use (10) to find the virtual coupling which makes the haptic interface absolutely stable, please refer to the authors previous work [15].

\section{EXPERIMENTS AND VALIDATION}

In order to test the accuracy and reliability of the proposed user interface system with haptics enabled simulation, we used experimental real data. The geometrical dimensions and the mechanical properties are determined through a microinjection setup [20]. The experimental data (shown in Fig.5) depicts the force and deformation measurement process on a mouse oocyte ZP. The forces increase nonlinearly as deformation increase. When the deformation reaches about $45 \mu \mathrm{m}$, the $\mathrm{ZP}$ and the plasma membrane are punctured, the puncturing forces are approximately $7.5 \mu N$ [20]. The experimental data are plotted with $95 \%$ prediction bounds (error bounds 5 \%) for more precision (Fig.5). The Fig.6 presents the error between experimental data and regression curve. In order to demonstrate the validity of the proposed real-time finite element method, we compared the simulation results of a needle insertion task using the physics-based FEM model with the experimental data provided by a real test carried out on a mouse oocyte [20]. The mechanical and geometrical properties used in the finite element simulations are settled in Table.I.

The Fig.7 presents the experimental data, the linear and non-linear finite element relationship between the needle insertion and the reaction force for mouse oocyte cells. The simulations show that the non-linear FE St-Venant-Kirchhoff 


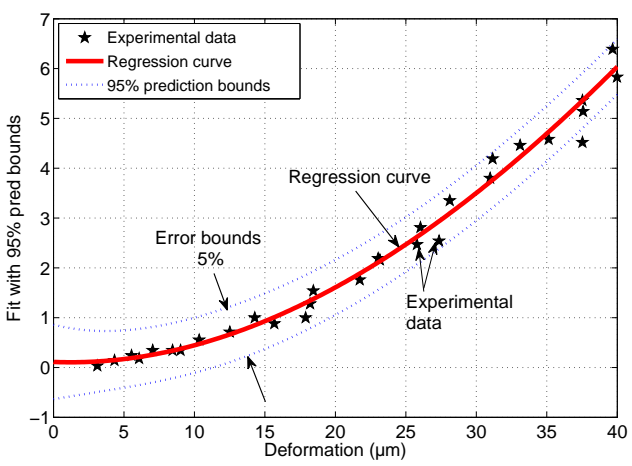

Fig. 5. Experimental data: force versus needle insertion deformation of the mouse oocyte cell with $95 \%$ prediction bounds.

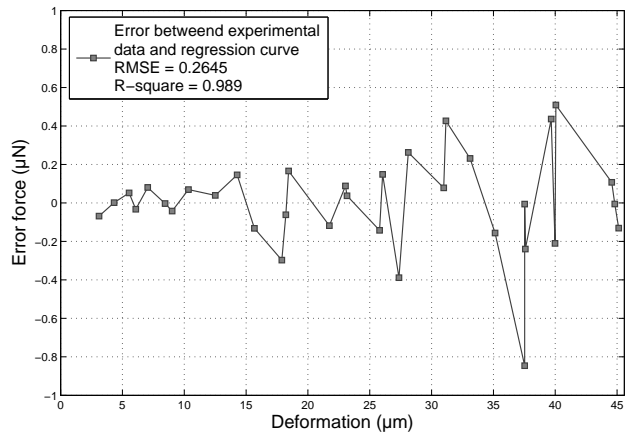

Fig. 6. Error between experimental data and regression curve.

model is in good agreement with experimental data. It can be clearly seen that the linear finite element model is valid only for small displacement (less than $10 \%$ of the mesh size).

TABLE I

Mechanical and Geometrical Properties of mouse oocyte.

\begin{tabular}{c|c}
\hline & Mechanical and Geometrical Properties \\
\hline \hline Young modulus & $17.9 \mathrm{KPa}$ \\
Poisson coefficient & 0.49 \\
Diameter & $56 \mu \mathrm{m}$ \\
The mass of the cell & $9.195210^{-5} \mathrm{mg}$ \\
Vertex mass & $0.0799510^{-5} \mathrm{mg}$ \\
Cell volume & $91952 \mu \mathrm{m}^{3}$ \\
Mesh of the cell & 115 vertices and $332 \mathrm{Tetra}$ \\
Maximum deformation & $40 \sim 44 \mu \mathrm{m}$ \\
\hline
\end{tabular}

\section{A. Evaluation}

An experimental system was implemented on a personal computer with a Intel Core Duo $3.2 \mathrm{GHz}$ CPU with 4 GB memory, and GeForce 8600 GTS with 512Mo memory. In this experiment, we investigated the time required for precomputation (off-line computation) and the real-time deformation process by changing the number of vertices of a cell model (Table.II). The relationship between the number of vertices and the off-line computation was identified by a nonlinear law or this relation between the number of vertices and realtime simulation was described by a linear law (see Fig.9).

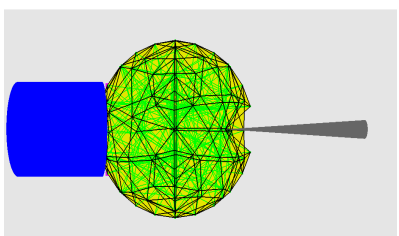

$10 \mu m$ needle insertion

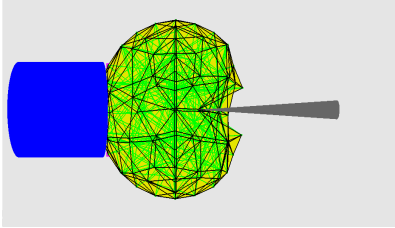

$20 \mu m$ needle insertion

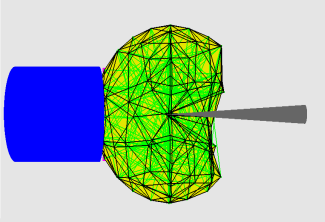

$30 \mu m$ needle insertion

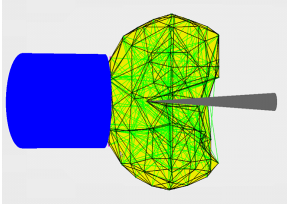

$40 \mu m$ needle insertion

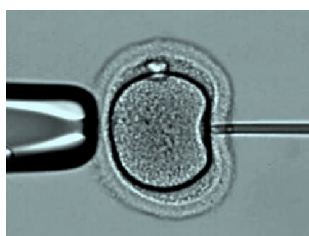

real picture

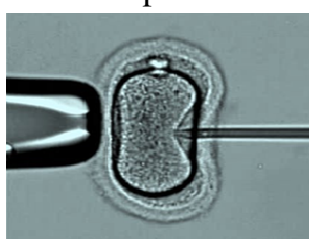

real picture

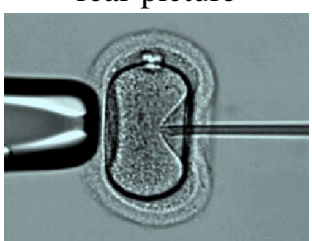

real picture

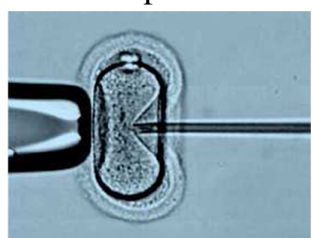

real picture
Fig. 7. Visual comparison of the various stages of deformation of needle insertion between FEM simulations and experimental data.

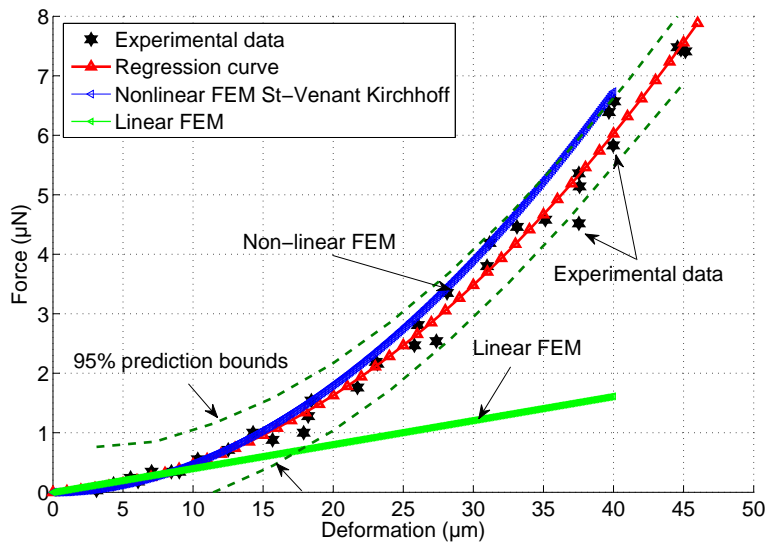

Fig. 8. Force versus deformation: comparison between experimental data, non-linear St Venant Kirchhoff and linear finite element simulations.

However, The accuracy and computational time of the finite element analysis depends on the number of the vertices and tetrahedron included in the mesh. The problem should be solved by using graphics processing unit (GPU) to perform finer mesh simulations.

\section{Conclusion}

We have developed a computer-based training system to simulate real-time cell micro-injection procedures in virtual 
TABLE II

COMPUTATION TIME VERSUS FINITE ELEMENT MESH REFINEMENT USING THE NONLINEAR MODEL

\begin{tabular}{cccc}
\hline \hline Vertices & Tetrahedron & $\begin{array}{c}\text { Pre-computation time } \\
\text { off-line }(\mathrm{ms})\end{array}$ & $\begin{array}{c}\text { simulation time } \\
\text { on-line }(\mathrm{ms})\end{array}$ \\
\hline 115 & 332 & 0.875 & 0.047 \\
123 & 379 & 0.99 & 0.047 \\
128 & 407 & 1.094 & 0.063 \\
165 & 546 & 1.718 & 0.078 \\
176 & 614 & 2.031 & 0.094 \\
192 & 672 & 2.359 & 0.109 \\
271 & 979 & 4.546 & 0.156 \\
282 & 1019 & 4.859 & 0.156 \\
346 & 1269 & 7.39 & 0.187 \\
516 & 2130 & 18.828 & 0.328 \\
731 & 3060 & 36.5 & 0.453 \\
1093 & 4819 & 88.734 & 0.703 \\
\hline
\end{tabular}

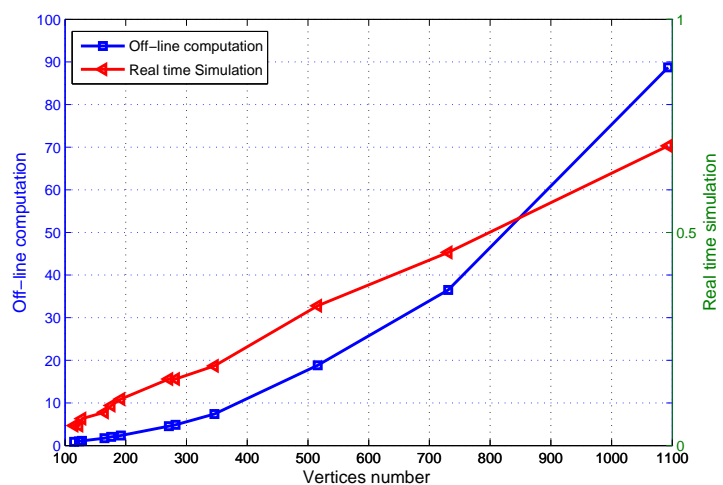

Fig. 9. Computation time versus vertices number: Pre-computation time and real-time simulation.

environments for training biologist residents. The simulator provides the user with visual and haptic feedback. The simulation of this procedure involves the real-time rendering, physically-based modeling of soft biological tissue, and display of touch and force sensations to the training through the simulation of haptic interactions. We first investigated the challenging issues in the real-time modeling of the biomechanical properties of the cell micro-injection through finite element models. Compared to experimental data performed on oocyte cells, we can see clearly that the proposed physicallybased FEM model is able to simulate the cell deformation through real-time simulation constraints. Currently, we are working on integrating others effects such as friction, viscosity, and adhesion forces, and the simulator is prepared to doing an experimental training evaluation from trained and non-trained candidates. All modalities will be merged in an ergonomic and intelligent biological simulator to support learning microinjection and training tasks.

\section{REFERENCES}

[1] P.M.M. Kastrop, S.M. Weima, R.J. Van Kooij, E.R. Te Velde, ”Comparison between intracytoplasmic sperm injection and in-vitro fertilization (IVF) with high insemination concentration after total fertilization failure in a previous IVF attempt", European Society of Human Reproduction and Embryology, vol.14 no.1 pp.6569, 1999.

[2] J.C.M. Dumoulin , E. Coonen, M. Bras , J.M. Bergers-Janssen , R.C. Ignoul-Vanvuchelen, L.C.P. van Wissen J.P.M. Geraedts, L.H. EVERS
Johannes, "Embryo development and chromosomal anomalies after ICSI effect of the injection procedure", Hum Reprod. 16, pp. 306-312, 2001.

[3] T. Ebner, C. Yaman, M. Moser, M. Sommergruber, K. Jesacher, G. Tews, "A prospective study on oocyte survival rate after ICSI influence of injection technique and morphological features", J Assist Reprod Genet 18, pp. 601-606, 2001.

[4] A. Pillarisetti, M. Pekarev, A. D. Brooks, J. P. Desai, "Evaluating the Effect of Force Feedback in Cell Injection", IEEE Transactions on Automation Science and Engineering, 2007, 4(3): pp. 322-331.

[5] J. Kim, C. Choi, S. De, M.A. Srinivasan, "Virtual surgery simulation for medical training using multi-resolution organ models", Int J Med Robotics Comput Assist Surg, 3, pp. 149-158, 2007

[6] R.M. Satava, "Surgical education and surgical simulation", World J Surg , 25(11), pp. 1484-1489, 2001.

[7] U. Meier, O. Lopez, C. Monserrat, M.C. Juan, M. Alcaniz, "Real-time deformable models for surgery simulation: a survey", Comput Methods Programs Biomed, 77(3), pp. 183-197, 2005.

[8] R. Alterovitz, K. Goldberg,"Planning for steerable bevel-tip needle insertion through 2D soft tissues with obstacles", in Proc. IEEE Int. Conf. on Robotics and Automation (ICRA), pp. 1652-1657, 2005.

[9] H.W. Nienhuys, A.F. vander Stappen , "A computational technique for interactive needle insertions in 3D nonlinear material", in Proc. IEEE Int. Conf. on Robotics and Automation (ICRA), pp. 2061-2067, 2004.

[10] N. Bolhassani, R. Patel, M. Moallem , "Needle insertion into soft tissue : A survey", Med Eng Phys, 29 (4), pp. 413-431, 2007.

[11] A. Pillarisetti, M. Pekarev, A.D. Brooks, J.P. Desai, "Evaluating the Effect of Force Feedback in Cell Injection", IEEE Transactions on Automation Science and Engineering, 4(3), pp. 322-331, 2007.

[12] M. Ammi, H. Ladjal, A. Ferreira, "Evaluation of 3D Pseudo-Haptic Rendering using Vision for Cell Micromanipulation", IEEE Intelligent Robots and Systems, Beijing, China, 2006, 2115 - 2120

[13] H. Ladjal, J.L. Hanus and A.Ferreira, "Interactive Cell Injection Simulation Based on 3D bio-mechanical Tensegrity Model", IEEE International Conference on Robots and Intelligent Systems (IROS'08), September 2226, 2008, Nice, France, Page 2296-2302.

[14] V. Lulevich, T. Zink, H-Y. Chen, F-T. Liu, G-y. Liu, "Cell Mechanics using Atomic Force Microscopy-based Single-Cell Compression", Langmuir, vol. 22, no. 19, 2006, pp. $8151-8155$.

[15] H. Ladjal, J.L. Hanus, A. Pillarisetti, C. Keffer, A.Ferreira and J.P. Desai,"Reality-based real-time Cell Indentation Simulator", IEEE/ASME Transactions on Mechatronics, 2010, DOI 10.1109/TMECH.2010.2091010.

[16] H. Delingette, N. Ayache, "Soft tissue modelling for surgery simulation", Computational Models for the Human Body, Handbook of Numerical Analysis, Elsevier, 2004.

[17] S. Cotin, H. Delingette, N. Ayache, "A Hybrid Elastic Model for RealTime Cutting, Deformations, and Force Feedback for Surgery Training and Simulation", Visual Computer 16, 2000, 437-452.

[18] J. Picinbono, H. Delingette, N. Ayache, "Non-linear and Anisotropic Elastic Soft Tissue Models for Medical Simulation". Proc, IEEE International Conference on Robotics and Automation, Seoul, Korea, 2001, $1370-5$.

[19] H. J. Bathe, "Finite Element Procedure", Prentice hall, New-York, 1996.

[20] Y. Sun, K. Wan, K.P. Roberts, J.C. Bischof, B.J. Nelson, "Mechanical property characterization of mouse zona pellucida", IEEE Transactions On NanoBioscience, Vol. 2, No. 4, 2003.

[21] T. Moller, B. Trumbore, "Fast, Minimum Storage Ray-Triangle Intersection", Journal of graphics tools, vol. 2, no. 1, pp.21-28, 1997.

[22] R. Adams, M. Moreyra, B. Hannaford, Stability and performance of haptic displays : theory and experiments, Proceedings of the ASME Winter Annual Meeting Haptics Workshop, Anaheim, USA, 1998.

[23] S. Yu, B.J. Nelson, "Microrobotic Cell Injection", IEEE International Conference on Robotics and Automation ,Seoul, Korea, 2001, 620-625.

[24] X. Liu, Y. Sun, W. Wang, B.M. Lansdorp, "Vision-based cellular force measurement using an elastic microfabricated device", Journal of Micromechanics and Microengineering, Vol.17, 2007, pp.1281-1288.

[25] F. B. Llewellyn, Some fundamental properties of transmission systems, Proceedings IRE, Vol. 40, 1952.

[26] O. Goksel, S. E. Salcudean1, S.P. Dimaio, "3D simulation of needletissue interaction with application to prostate brachytherapy", Computer Aided Surgery, Vol. 11, No. 6, , Pages 279-288, 2006

[27] O. Goksel, S. E. Salcudean1, S.P. Dimaio, "Needle insertion modeling and simulation", IEEE Transactions on Robotics and Automation, Vol. 19 Issue:5, Pages 864-875, 2003. 